\title{
SIMULATING CORTICAL MAPS FOR ATTENTION SHIFT IN AUTISM
}

\author{
L.-H. Tan, S.-Y. Cho and Y.-Y. Nguwi \\ School of Business (IT), James Cook University, Singapore
}

\begin{abstract}
Autism is a pervasive neuro-developmental disorder, primarily encompassing difficulties in the social, language, and communicative domains. Because autism is a spectrum disorder, it affects each individual differently and has varying degrees. There are three core aspects of impairment based upon the Diagnostic and Statistical Manual of Mental Disorders (DSM-IV), namely impairment in socialization, impairment in communication, and restricted repetitive activities or interests. This work describes the experiment aims at expressing autistic traits through the use of self-organizing map. Works related to simulating autism through self-organizing map is limited. This work compare and contrast the difference in attention index for normal learning and marred attention shift learning ability. It was found that the attention index of normal learning is 9 times better marred attention shift for both random and pre-fixed input data. In the marred attention shift context, neurons adapt more towards the mean of both sources combined under marred context while some neurons adapt towards mean of one source under normal context. The normal learning ability produces maps with neurons orienting towards mean values of combined stimuli source. Impairment in learning ability produces similar cortical maps compared to normal learning ability. The major difference is in the attention index.
\end{abstract}

\section{KEYWORDS}

self-organizing map, attention shift, autism, neural network

\section{INTRODUCTION}

Autism is a complex neurological illness characterized by intricate defects mainly in three aspects: communication, social interaction and imagination [1]. The disease was initially described by Kanner [2] and Asperger [3]. Asperger used the term autism to define the crux elements of the disease. Inability to carry out normal nonverbal and verbal communication, familiarity preference, extreme autistic loneliness and restricted repertoire of actions were four basic traits of Autism examined by Kanner. In 1978, the idea of child autism as a prominent neurological basic trait contributed to the commencement of research studies, clinical speculation and progression of primary precise operationally diagnostic requirements $[4,5]$.

As many as 1.5 million Americans today are believed to have some form of autism, with many more having related pervasive developmental disorders (PDD), such as Asperger's disorder (AD) [6]. Because autism is a spectrum disorder, it affects each individual differently and has varying degrees. One model of autism, the weak central coherence account, addresses the fact that people with autism often focus on component parts rather than wholes [7]. As a result, people with autism often show superior performance on some visually presented tasks and other tasks that favour such focus. However, tasks that require integration or discrimination among many sensory stimuli or abstract concepts can be difficult to autistic patients. This can include computationally heavy tasks such as envis ioning another's state of mind in social settings or imaging future states

DOI: $10.5121 /$ ijcnc.2016.8405 
sufficiently to plan a task [8]. This can also put people with autism at risk for sensory overload, and difficulty in functioning in the presence of many visual or auditory distracters.

Neuroanatomical studies [9] have shown that abnormal growth of brain are observed in individuals suffered from autism. Such features were also observed in the magnetic resonance imaging (MRI) studies by Courchesne et. al [10, 11]. It was demonstrated that significant decrease in Purkinjie cells presence in cerebellum is frequently detected in autism brain [12] as well. In addition, attention deficit, weak eye contact, known as "savant skills", sensory hypersensitivities and hyposensitivities, stereotyped ritualistic behaviours, weak generalization means, perseveration, hyperspecificity and attention to details rather than the whole $[13,14]$ were some of the attributes associated with the illness as well. Mental retardation is the most commonly associated trait of autism. In fact, a lack of eye contact is the most common feature of autism and further confirmations are given in eye tracking studies [15] showing decreased attention on the eyes and increased attention on the mouth or surrounding environment.

There are three core aspects of impairment [1] based upon the Diagnostic and Statistical Manual of Mental Disorders (DSM-IV) are namely impairment in socialization, impairment in communication, and restricted repetitive activities or interests.

Social difficulties may present in autistic individuals due to low levels of vasopressin, a neuropeptide found in the brain. In a studies by Parker et. al., they begun a clinical trial to treat individuals with vasopressin to reduce their social problems. The experiments demonstrated improved social cognition and memory are observed in people who do not have autism [16]. Autistic individuals display weak intercommunication abilities which tend to be stilted and mechanical when they do happen [17]. Children with autism are not able to establish and maintain cooperation and social relationships due to the difference in their usages and comprehension of gestures for socialization with others $[1,18]$. Autism individuals were observed to display severe social avoidance behaviour [19].

The second core trait of autism is the impairment in communication. Autistic individuals have difficulties in understanding figurative language concepts [20]. They interpret spoken conversation directly [21] and tend to reiterate what others said to them. Hermelin [22] discovered that autistic child was incompetent of recoding details from sensory to abstract codes. Consequently, people with autism are incapable of viewing and deciphering abstract and salient elements of a scenario [7] compared to normal individuals. Moreover, the language used in autistic individuals is mostly made up of needs requests rather than descriptive interaction [18]. It is vital to explore this trait with the use of cortical map demonstrating these impairments in autistic individuals for better understanding and counter-measure.

The third trait is restricted repetitive of activities or interests. Exhibition of restricted repetitive of activities and interests were commonly observed in autism. Dawson et al. [23] found that autistic children displayed attention shift deficits in the presence of social stimuli while Pascualvaca et al. [12] discovered that people with autistic do not have general hindrance in shifting attention. Moving on, Dawson and Lewy [24] realised that aversive reaction may arise when novelty is introduced. People with autism are hypothesized to be compulsive and obsessive with the current state of predicament they are in. Kootz et al. [25] also found out that child with autism response to novelty with avoidance.

This paper is organized as follows: Section 2 provides background studies of self-organizing map. Section 3 describes the use of self-organizing map for Autism Simulation, citing the related works in this area. In section 4, we presents a framework for autism simulation. The framework includes involve identifying simulating features, obtaining optimal base parameters, creating different 
International Journal of Computer Networks \& Communications (IJCNC) Vol.8, No.4, July 2016

stimuli context, fine-tuning of cortical map, computation of attention shifts, and evaluating analysis. Section 5 discusses the maps formation. Finally, conclusion is drawn in section 6 .

\section{SELF-ORGANIZING MAP BACKGROUND}

Self-Organizing Map learns in an unsupervised fashion without feedback from a teacher. It is extremely useful in visualizing data of high dimensionality using low dimensions. The neurons go through competitive learning. An output neuron that wins the competition is called the winning neuron. The goal of SOM is to transform an incoming signal pattern of arbitrary dimension into a one- or two- dimensional discrete map, and to perform this transformation adaptively in a topologically ordered fashion.

Kohonen [26] describes SOM as a nonlinear, ordered, smooth mapping of high-dimensional input data manifolds onto the elements of a regular, low-dimensional array. Assume the set of input variables $\left\{\xi_{j}\right\}$ is definable as a real vector $x=\left[\xi_{1}, \xi_{2}, \ldots, \xi_{n}\right]^{T} \in \Re^{n}$. With each element in the SOM array, we associate a parametric real vector $m_{i}=\left[\mu_{i 1}, \mu_{i 2}, \ldots, \mu_{i n}\right]^{T} \in \mathfrak{R}^{n}$ that we call a model. Assuming a general distance measure between $x$ and $m_{i}$ denoted $d\left(x, m_{i}\right)$, the image of an input vector $x$ on the SOM array is defined as the array element $m_{c}$ that matches best with $x$, i.e., that has the index

$$
c=\arg \min _{i}\left\{d\left(x, m_{i}\right)\right\}
$$

The task is to define $m_{i}$ in such a manner that the mapping is ordered and descriptive of the distribution of $x$. The data points that are projected to close-by locations on the map are close-by also in the input space. The ability of self-organizing according to neuron's neighbourhood Euclidean distance is the key feature of SOM.

There are two types of maps [27, 28]: the cortical maps and maps of ocular dominance. Cortical maps correspond to line association. Ocular dominance map denotes the influence of electrical signal imposed on eyes. Both maps reveal the presence of hierarchical structure of somatosensory, auditory, and visual maps in human brain. Self-organizing map can be applied in a supervised or unsupervised manner. Unsupervised learning takes place when stimuli of the same clusters and output activate the nodes spatially on the map. It produces outputs that enable us to view the relationship exists among clusters in cortical maps.

\section{Self-Organizing MaP fOR AUtism Simulation}

Self-Organizing Map (SOM) can be used to model biological sensory areas by modifying input stimuli encoded in the topology of SOM. The behaviours of autistic and normal persons in the focus-attention and shift-attention tasks were depicted in [18]. Generalization in autistic children may be due to predispose of a form of neural network that disable them from manipulating overlaps [29]. There are a number of different ways to explain autism such as increased neurogenesis, decreased neuronal call death, abnormal myelin, decreased synaptic pruning and increase production or non-neural tissues were made with neurobiological techniques which require further exploration [30].

Autistic individuals are discovered to have the inability in recoding information from sensory to abstract codes by Hermelin [22]. In turn, viewing features regarded as salient to normal children 
International Journal of Computer Networks \& Communications (IJCNC) Vol.8, No.4, July 2016

were a challenge to autistic individuals [31]. An inadequate SOM, where correct grouping of stimuli may not be completed, may be created during the learning process for a neural network. Autism could be modelled as a consequence of ill-developed and highly discriminative cortical SOM [32]. Apart from that, the familiarity preference in stimulus selection can also be shown as a result of deficient SOM characteristics [33, 34].

Lennart et. al [35] investigated the effects of attention shift impairment and familiarity preference in self-organizing map. Developed maps were discussed in details. The experiments demonstrated that familiarity preference results in inadequate maps with characteristic impairments, supporting the hypothesis of novelty avoidance being the primary reason of other autistic features.

Gerardo et. al. [36] examined abnormalities in neural development in the brain of autistic children, sensory issues, the ability of generalization and the effects of noise communication routes between neurons on the maps generated. The performance of SOMs generated was measured in terms of effectiveness of stimuli coverage and map topology. Inferences proposed in the work were supported by previous statistical analyses. Modelling of abnormal neural growth in autism was done by manipulating the dimensions of the SOM-based network. A distinct negative impact was observed in the unfolding of the neural network which changes with the intensity of the aberrations. To analyze sensory issues, concept of attention functions was introduced. While modelling hypersensitivity, it was deduced that an excess in specific aspects of excellent aptitude may cause the dismissal of input to the hypersensitive domain. The authors [36] managed to reproduce the tendency of autism personnel to concentrate on details than the whole. During the process, it was stated that noise does not aid to explain the autism characteristic of focus to details.

\section{FRAMEWORK FOR AUTISM SIMULATION}

In this section, we will discuss the framework for autism simulation. The steps (as depicted in Figure 1) involve identifying simulating features, obtaining optimal base parameters, creating different stimuli context, fine-tuning of cortical map, computation of attention shifts, and finally evaluating analysis.

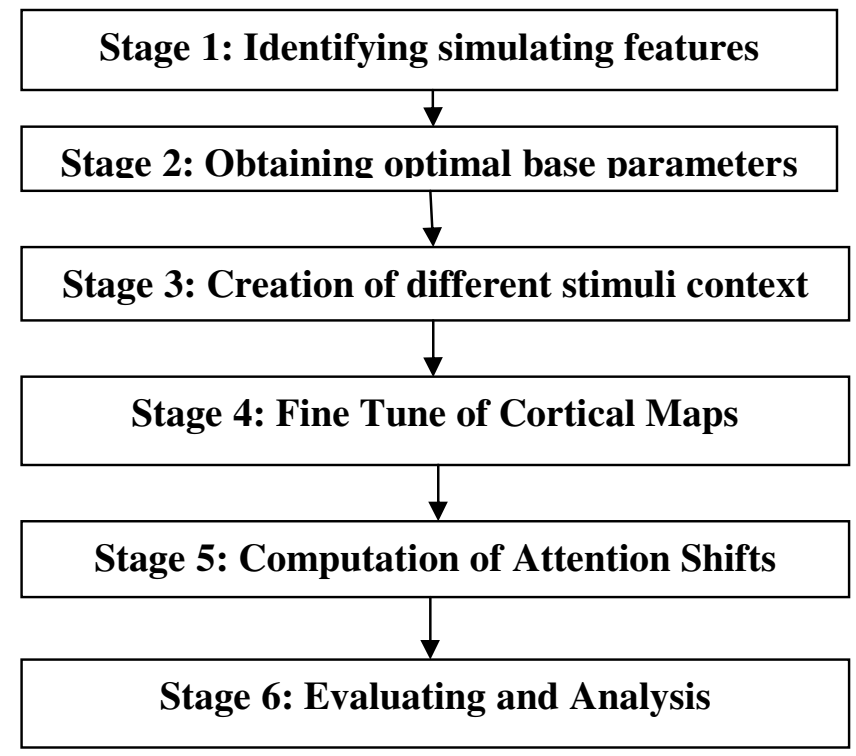

Figure 1 Framework for Autism Simulation 
Stage 1 finds out the features appropriate for autism traits. We focus on the traits of novelty avoidance (familiarity inclination) and concentration impairment.

In stage 2, we evaluate the most appropriate parameters values essential and suitable for cortical map formation. The input data is formatted and processed with appropriate training length, learning rate, and neighbouring function. We adopted two sets data input, first data format is randomized while the second set of data is made up of pre-fixed inputs. Each set of data inputs represent different source of provocation for displaying the interaction of neurons when presented with different stimuli under dissimilar conditioned context during simulation. Training length affects the cortical maps formation and map convergence issue. Different neighbouring functions were tested like Bubble function, Gaussian function, Cut Gaussian function, and Epanechicov function [22, 24].

Stage 3 involves the creation of context. We simulate three kinds of context: namely normal learning, marring of learning aptitude and familiarity inclination [29]. In normal learning, neurons adjust themselves towards new inputs in the maps concocted out. The competitive learning behaviour of Self-Organizing map imitate the normal reaction expected when new stimuli is perceived from the environment. In marring of learning aptitude, impairment of learning ability in autism was formulated by changing the learning parameter. Reduction of learning parameter consequently reduces neurons learning speed in the simulation, replicating the tendency of autistic individuals who react slower or otherwise indifferently to novelty. The marring of learning aptitude learning uses two data sets. The first data sets allows for neurons to adapt first, then second data set is added as new source. The neurons will then be adapted more towards the first dataset. The probability of association towards the second data set is greatly hindered by the restricted fixed learning rate. It would take longer training time to achieve similar output than normal learning mode. In familiarity inclination context, two steps are involved. Firstly, it takes the output from normal learning mode and uses it in impairment of learning mode to create familiarity inclination. Familiarity is achieved by averaging the distance between weights of nodes closest to the source already existed.

Stage 4 fine tunes the generated maps. Upon completion of stage 1 and 2, cortical maps obtained needed to be fine tuned for easy readings and better defined clusters. To do so, we could try several ways introduced by Kohonen in his articles on Self-Organizing Maps [26]. Instead, simulation of each different mode was done repetitively for at least fifty times till consistent results is observed to abstract desired maps. Having repetitive simulation compensates the minimum training length applied. Higher training length would give us better maps. However, as stated in earlier section, training length was kept to the minimum to enhance efficiency of experimental progress. Each output is measured and compared with those collected in [35].

Stage 5 is the computation of attention shifts. Attention shift calculated from maps generated were the focus of this experiment. Input data set and neurons output position on generated maps were extracted. Mean differences between the coordinates were computed. Average values of each axis point were set as the combined average mean differences calculated. With the average values computed, standard deviation is applied. Results formulated were multiply by a thousand. Comparison between $\mathrm{X}$ and $\mathrm{Y}$ standard deviation were carry out and consistent largest value is assigned as attention shift required for evaluation.

Stage 6 evaluates the analysis. The last stage of this experiment design involves comparing and analysing of outputs constructed in stage 4 and 5 with cortical maps studied in [35]. Similar maps were retrieved and attention shifts for each identified map were calculated. 


\section{MAPS FORMATION}

This work was implemented using Matlab, in particular the SOM toolbox [37]. Two sources of data are adopted, source 1 (using randomised data) and source 2 (using adjusted input data). Each simulation comprises of approximately 100 data inputs.

Figure 2 displays the trained map using (i) random data and (ii) adjusted data. The neurons (blacked dots) scatters evenly around data (red x). Neurons adaptation or association appear to be insignificant. Thus the need for using pre-fixed adjusted input data. The adjusted input distributes around four corners. It can be observed from the figure that neurons adapt itself towards data matrix moving towards the corner.

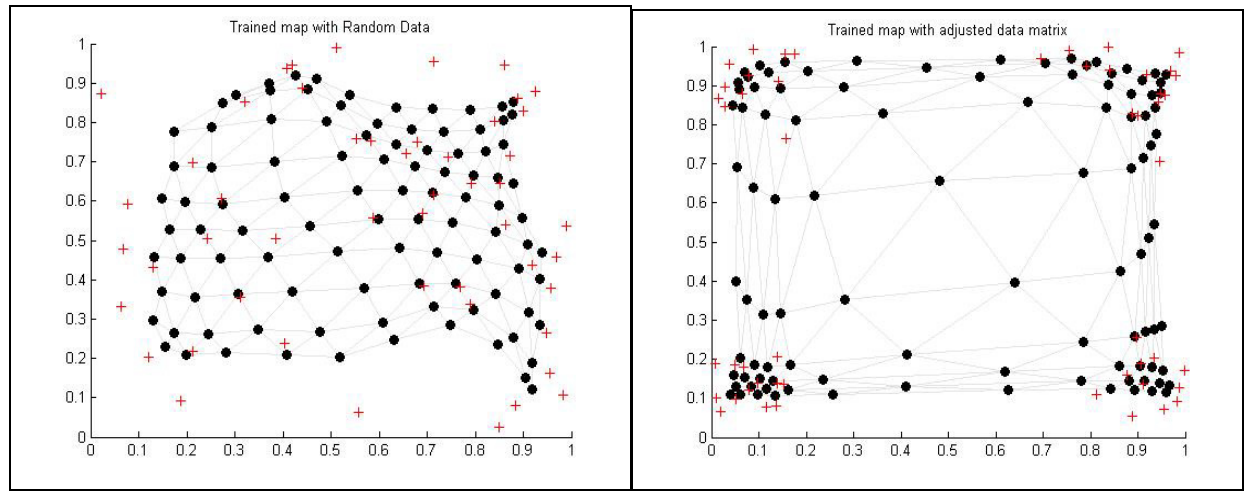

Figure 2 Trained map with (i) Random data

(ii) Adjusted data

Figure 3 presents the map with (i) 1000 epoches and (ii) 10,000 epoches. The 10 times longer training time does not really give rise to differentiated map. For training time beyond 10,000, the training appears to slow down significantly and has problem converging.

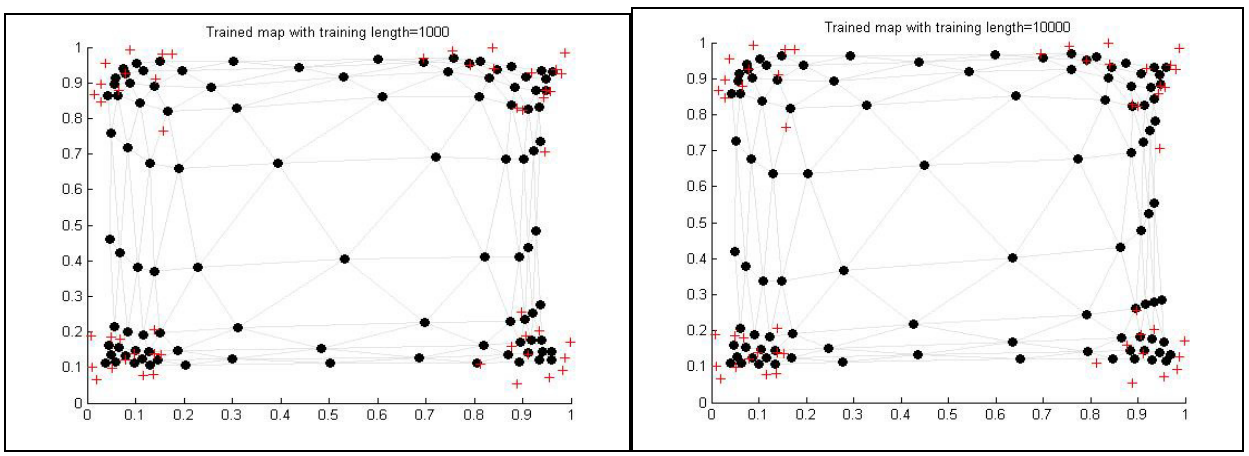

Figure 3 Trained map with (i) 1000 epoches

(ii) 10,000 epoches

Figure 4 depicts maps with different learning rate from 0.1 to 100 . Learning rate controls how well neurons associate themselves to input data within a given period. Low learning rate may impede growth of association among neurons. While high learning rate is advisable, learning rate might still reach a saturation point where further increments of learning value fails to produce better maps. Overall, appropriate higher learning rate construes well defined cortical maps. Various learning rate were applied. The first range employs values between 0.01 and 0.1 to see the impact of setting small initial rate. After realizing the impact of low learning rate, 0.1 to 1.0 were further investigated to discover the minimum rate for a norm. The last range of studied 
International Journal of Computer Networks \& Communications (IJCNC) Vol.8, No.4, July 2016

values includes numbers from 1.0 to 10 and 100 to find out the optimal applicable optimal rate. The first training graph (Figure 4(i)) resembles an X shape. The learning rate is too low to obtain the respective cortical map. The second map generated Figure 4(ii) shows significantly better "learnt" map. The map with learning rate of 100 (Figure 4 (iii)) shows slightly improved map compare to Figure 4(ii). Saturation occurs when learning rate is 10.

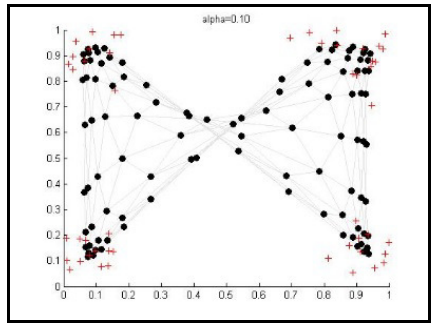

(i) Learning rate $=0.1$

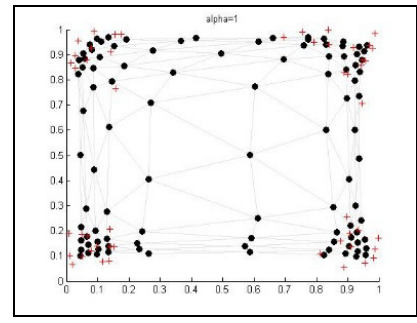

(ii) Learning rate $=1.0$

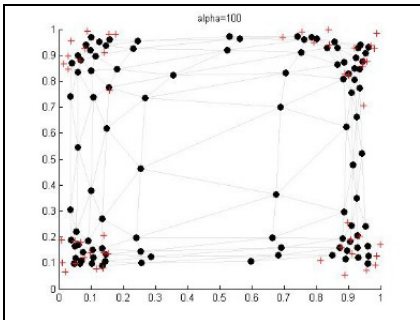

(iii) Learning rate $=100$

Figure 4 Trained map with varying learning rate

The previous section (Section 4) discussed the three contexts in use, namely normal learning, marring of learning aptitude formation, and familiarity inclination. We formulate the learning as follows:

Table 1 Normal Learning Formulation

Steps for (I) Normal Learning Formulation

1. Construct two separate matrix data input representing two sources.

2. Fine-tune the right learning rate, training length, and neighbouring function.

3. Label neurons and input sources.

4. Maps slowly forms up until consistent construct is observed.

Table 2 Marring of Learning Aptitude Formulation

Steps for (II) Marring of Learning Aptitude Formulation

1. Construct two separate matrix data input representing two sources.

2. Fine-tune the right learning rate, training length, and neighbouring function.

3. Set the learning rate to be 0.3 to impede learning ability

4. Label neurons and input sources.

5. Maps slowly forms up until consistent construct is observed.

Table 3 Familiarity Inclination Formulation

Steps for (III) Familiarity Inclination Formulation

1. Construct two separate matrix data input representing two sources.

2. Fine-tune the right learning rate, training length, and neighbouring function.

3. Set the learning rate to be 0.3 to impede learning ability.

4. Label neurons and input sources.

5. Generate rate the map with one source of data.

6. Maps slowly forms up until consistent construct is observed.

7. Second source of data is added to existing map obtained in 5. Repeat step 6 until the map converge. 
International Journal of Computer Networks \& Communications (IJCNC) Vol.8, No.4, July 2016

Attention index depicts the ability of normal and autistic person to relate to novelty when new stimuli are introduced. Higher value of attention index implies higher adaptability. Attention index is calculated based on the sum of differences of $x-y$ coordinates of input and neurons, followed by multiplying thousand to its standard deviation.

We arrange the data into 2 batches: first batch being a 3 by 3 mesh, second batch with lesser data incorporated for one source. Figure 5 displays the cortical maps formed after training. The first batch result shows two different input source as represented by red circle and blue cross. Attention index for first batch is found to be 61190. The second batch simulates the map by having one of its input source halved in number. The resultant map shows indifferent neurons pattern and attentions index is also 61190. This shows that the learning ability of neurons in a normal neural system is not compromised despite lesser information is received from new stimuli.

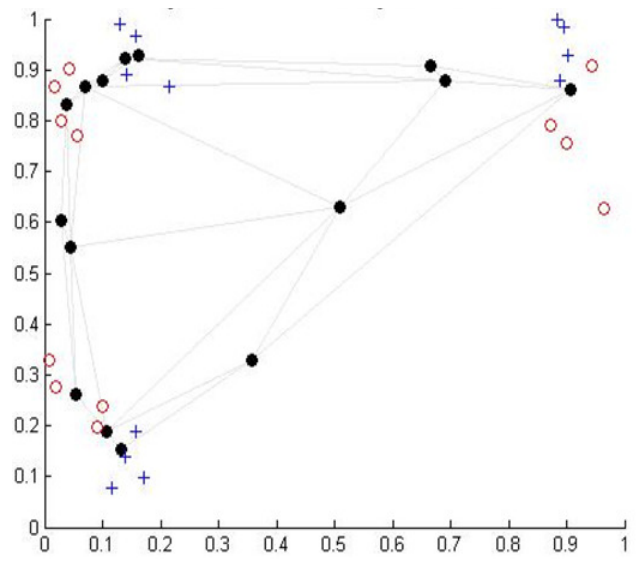

(i) First Batch

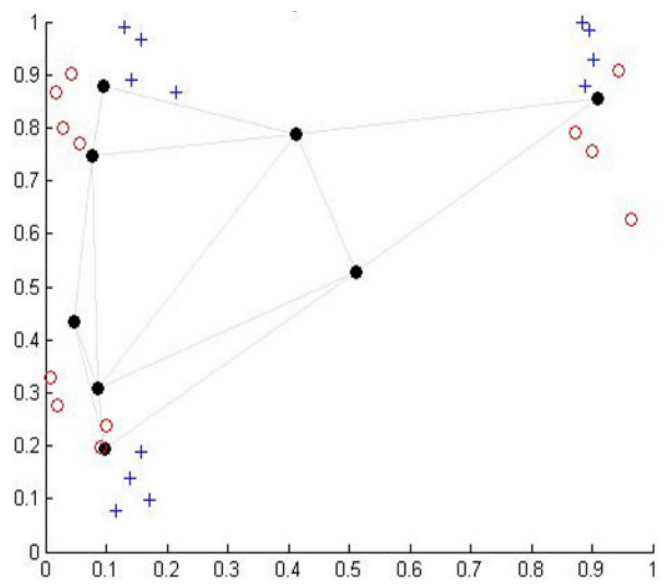

(ii) Second Batch

Figure 5 Cortical maps for normal learning context

Figure 6 presents the cortical map for marred attention shift learning ability. It is observed that the cortical output remain similar to normal learning context. The nodes adapts relatively better to mean results of the input source. Thus, when presented with an alternative source, the neurons will try to adapt to its means. This results in better contrast and node weight adjustment. The attention index obtained is 6495 for first batch. The first batch appears to have similar result as first batch. A closer look will reveal that neurons are more consistent in their adaptation compared to normal learning condition. Neurons adapt more towards the mean of both sources combined under marred context while some neurons are viewed to adapt towards mean of one source under normal context. The second batch also has the same attention index of 6495. It is noted that this drastic fall in attention shift compared to normal learning context is the basis of this work where attention shift is lesser in marred learning compared to normal learning. The attention index of normal learning (61190) is 9.4 times of marred attention shift learning (6495). 


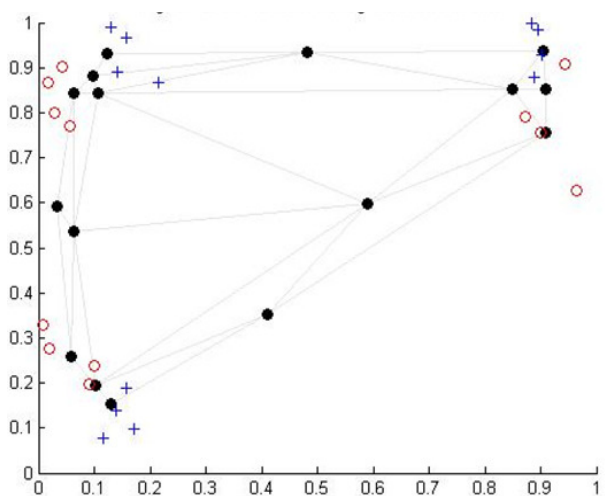

(i) First Batch

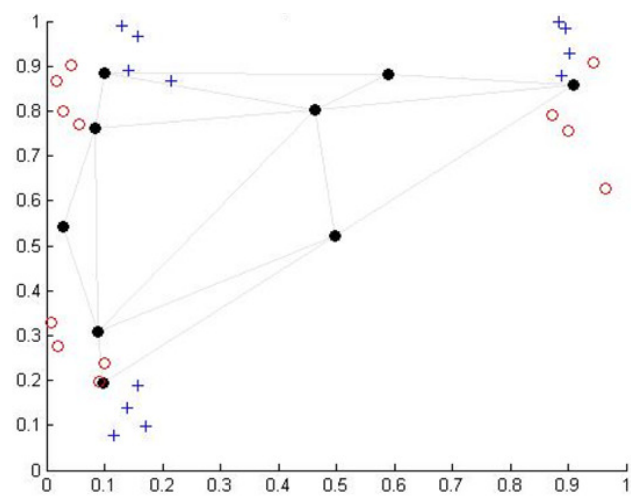

(ii) Second Batch

Figure 6 Cortical maps for marred attention shift learning ability

\section{CONCLUSIONS AND FUTURE TRENDS}

This paper described the experiment aims at expressing autistic traits through the use of selforganizing map. The common features of autistic traits were discussed, namely socialization, impairment in communication and restricted repetitive of activities and interest. Works related to simulating autism through self-organizing map is limited. This work compare and contrast the difference in attention index for normal learning and marred attention shift learning ability. It was found that the attention index of normal learning is 9 times better than marred attention shift for both random and pre-fixed input data. In the marred attention shift context, neurons adapt more towards the mean of both sources combined under marred context while some neurons adapt towards mean of one source under normal context. The normal learning ability produces maps with neurons orienting towards mean values of combined stimuli source. Impairment in learning ability produces similar cortical maps compared to normal learning ability. The major difference is in the attention index. Similarities of maps obtained from marred and normal learning ability can be explained by assuming neurons adapting themselves towards the mean of existing source produce greater node weight changes that pull the nodes nearer to mean value of combined new and existing sources.

\section{REFERENCES}

[1] American Psychiatric Association (2000). Diagnostic and statistical manual of mental disorders: text revision (4thed). Washington, DC: Author.

[2] L.Kanner," Autistic disturbances of affective contact," Nervous child, (2):217-250,1943.

[3] H.Asperger. Die "Autistischen Psychopathen' im Kindesalter. Arch.Psychiatrie Nervenkrankheiten," (117):76-136,1944. Translated in Frith U.(ed)(1991): Austism and Asperger Syndrome. Cambridge University Press.

[4] Ritvo E., Freeman B.(1978), "National Society for Autistic children definition of the syndrome of autism. J Autism Childhood Schizophrenia," 8:162-169.

[5] Rutter M.(1978), "Diagnosis and definition of childhood autism", J Autism Childhood Schizophrenia,8:139-384.

[6] LoPresti, E. F., Bodine, C., \& Lewis, C. (2008). Assistive technology for cognition [Understanding the Needs of Persons with Disabilities]. Engineering in Medicine and Biology Magazine, IEEE, 27(2), 29-39.

[7] Happe, F., Briskman, J., \& Frith, U. (2001). Exploring the cognitive phenotype of autism: Weak "central coherence" in parents and siblings of children with autism: I. Experimental tests. Journal of Child Psychology and Psychiatry and Allied Disciplines, 42(3), 299-307. 
International Journal of Computer Networks \& Communications (IJCNC) Vol.8, No.4, July 2016

[8] Hart, M. (2005). Autism/Excel study. Paper presented at the ASSETS 2005 - The Seventh International ACM SIGACCESS Conference on Computers and Accessibility.

[9] M.LBauman and T.L.Kemper, "Neuroanatomic observations of the brain in autism: A review and future directions," Int.J.Devl.Neurosci.,vol.23, no.2-3, pp.183-187,2005.

[10] E.Courchense.R-A.Muller, and O.Saitoh, "Brain weight in autism: Normal in the majority of cases, megalencephalic in rare cases," Nurology, vol.52. pp. 1057-1059, March 1999.

[11] E.Courchense, "Unusual brain growth patterns in early life patients with autistic disorder: An MRI study," Neurology, vol.57, pp.245-254, Jul.2001.

[12] D.M.Pascualvaca, B.D.Fantie, M.Papageorgiou, and A.F.Mirsky. Attentional capacities in children with autism: Is there a general deficit in shifting focus? Journal of Autism and Developmental Disorders, 28(6):467-478, 1998.

[13] F.R. Volkmar, R.Paul, A.Klin, and D.Cohen, " Handbook of Autism and Pervasive Developmental Disorders", 3rd Edition. New York:Wiley, April 2005.

[14] U. Frith, "Autism: Explaining the Enigma," 2nd edition. Oxford, U.K:Blackwell, April 2003.

[15] Falck-Ytter, T., et al. (2013). "Eye tracking in early autism research." Journal of Neurodevelopmental Disorders 5(1): 1-13.

[16] Carson DS, Garner JP, Hyde SA, et al. Arginine Vasopressin Is a Blood-Based Biomarker of Social Functioning in Children with Autism. PLOS One. 2015.

[17] Gladys Pollack. The Defining Characteristics of Autism. Reader's Digest, September 2001.

[18] Christain Balkenius, Luc Berthouze, Petra Bjorne. "A model of attentional impairments in autism: first steps toward a computational theory." Lund Unversity Cognitive Science, Kungshuset, Lundagard, S-222 22 Lund, Sweden

[19] R.L Koegel , K Dyer and L K Bell, “The influence of child-preferred activities on autistic children's social behavior," J Appl Behav Anal. 1987 Fall; 20(3): 243-252.

[20] Distinct Characteristics of Autism. Website:http://www.rimland.org/characteristics.htm

[21] The National Autistic Society. Website: http://www.autism.org.uk

[22] B.Hermelin. Images and language. In M.Rutter and E.Schoppler, editors, Autism: A Reapprasial of Concept and Treatment, pages 141-154. Plenum, New York, 1978.

[23] G.Dawson, A.N.Meltzoff, J.Osterling, J.Rinaldi, and E.Brown. "Children with autism fail to orient to naturally occurring social stimuli." Journal of Autism and Developmental Disorders, 28(6):479-485, 1998.

[24] G.Dawson and A.Lewy. Arousal, attention and the socioemotional impairments of individuals with autism. In G.Dawson, editor, Autism: Nature, diagnosis and treatment, pages 49-74. Guilford, New York, 1989.

[25] J.P.Kootz, B.Marinelli, and D.J.Cohen. Modulation of response to environmental stimulation in autistic children. Journal of Autism and Developmental Disorders, 12(2):185--193,1982

[26] Kohenen, T., "Self-Organizing Map", Proceedings of the IEEE Volume 78, Issue 9, Sept. 1990 Pages(s): 1464-1480.

[27] Satish Kumar, Neural Networks: A classroom approach, International Edition, Mc Graw Hill, 2000.

[28] Simon Hakin, Neural Network: A comprehensive Foundation, International Edition, 2nd edition, Prentice Hall

[29] J.L.McClelland, "The basis for hyperspecificity in autism: A preliminary suggestion based on properties of neural nets," J.Austism Developmental Disorders, vol.30, no.5, pp. 497-502, Oct.2000.

[30] M.LBauman and T.L.Kemper, "Neuroanatomic observations of the brain in autism: A review and future directions," Int.J.Devl.Neurosci.,vol.23, no.2-3, pp.183-187,2005.

[31] Sherpherd, G.M., and C.Koch, 1990. "Introduction to synaptic circuits," in The Synaptic Organiztion of the Brain, G.M.Sherperd, ed., pp.3-31. New York: Oxford University Press.

[32] Luis Alfredo Vidal de Carvalho, Nivea de Carvalho Ferreira, Adriana Fiszman, Av.Venceslau Bras and Rio de Janeiro, "A neurocomputational model for autism," in Proc.IV Brazilian Conf.Neural Networks, Jul.1999, pp.344-349.

[33] L.Gustafsson and A.P.Paplinski, "An attempt in modelling autism using self-organization maps," in Proc. Int. Conf. Neural Information Process., Nov 2002, pp. 1815-1818

[34] L.Gustafsson and A.P.Paplinski, "Self-organization of an artificial neural network subjected to attention shift impairments and familiarity preference, characteristic studied in autism," J.Autism Developmental Disorders. vol.34. no.2. pp. 189-198. Apr.2004.

[35] Lennart Gustfsson and Andrew P.Paplinski. An Experiment in Modelling Learning in Autism Using Self-Organizing Artificial Neural Networks, April 8, 2002 
International Journal of Computer Networks \& Communications (IJCNC) Vol.8, No.4, July 2016

[36] Gerardo Noriega, Member, IEEE. Self-Organizing Maps as a Model of Brain Mechanisms Potentially Linked to Autism

[37] Juha Vesanto, Johan Himberg, Esa Alhoniemi and Juha Parhankangas. Self-organizing map in Matlab: the SOM toolbox.Proceeding of the Matlab DSP Conference 1999, Espoo, Finland, November 16-17, pp. 35-40, 1999. 